\title{
Gender-related mortality for HIV-infected patients on highly active antiretroviral therapy (HAART) in rural Uganda
}

This article was published in the following Dove Press journal:

International Journal of Women's Health

II April 2010

Number of times this article has been viewed

\author{
Arif Alibhai ${ }^{1}$ \\ Walter Kipp' \\ L Duncan Saunders' \\ Ambikaipakan Senthilselvan' \\ Amy Kaler ${ }^{2}$ \\ Stan Houston ${ }^{3}$ \\ Joseph Konde-Lule ${ }^{4}$ \\ Joa Okech-Ojony ${ }^{5}$ \\ Tom Rubaale ${ }^{5}$ \\ 'Department of Public Health \\ Sciences, ${ }^{2}$ Department of Sociology, \\ ${ }^{3}$ Department of Medicine, University \\ of Alberta, Edmonton, Canada; ${ }^{4}$ School \\ of Public Health, Makerere University, \\ Kampala, Uganda; ${ }^{5}$ Kabarole Health \\ Department, Fort Portal, Uganda
}

Correspondence: Arif Alibhai

Department of Public Health Sciences,

3-50G University Terrace, University of

Alberta, Edmonton, Alberta, T6G 2T4,

Canada

Fax +I 7804920364

Email arif.alibhai@ualberta.ca

\begin{abstract}
The purpose of this study was to examine gender differences in mortality for human immunodeficiency virus (HIV) patients in rural Western Uganda after six months of highly active antiretroviral therapy (HAART). Three hundred eighty five patients were followed up for six months after initiating HAART. Statistical analysis included descriptive, univariate and multivariate methods, using Kaplan-Meier estimates of survival distribution and Cox proportional hazards regression. Mortality in female patients (9.0\%) was lower than mortality in males (13.5\%), with the difference being almost statistically significant (adjusted hazard ratio for females $0.55 ; 95 \%$ confidence interval [CI]: $0.28-1.07 ; P=0.08$ ). At baseline, female patients had a significantly higher CD4+ cell count than male patients (median 147 cells $/ \mu \mathrm{L}$ vs 120 cells $/ \mu \mathrm{L} ; P<0.01$ ). A higher CD4+ cell count and primary level education were strongly associated with better survival. The higher CD4+ cell count in females may indicate that they accessed HAART services at an earlier stage of their disease progression than males. A borderline statistically significant lower mortality rate in females shows that females fare better on treatment in this context than males. The association between lower mortality and higher CD4+ levels suggest that males are not accessing treatment early enough and that more concerted efforts need to be made by HAART programs to reach male HIV patients.
\end{abstract}

Keywords: antiretroviral treatment, gender, rural, Uganda

\section{Introduction}

Globally, gender differences in life expectancy have been well documented with life expectancies being generally higher for females than for males. ${ }^{1}$ The life expectancy gap is typically much narrower in developing countries compared to developed countries. For example life expectancy in less developed countries (following conventions used by the United Nations) is on average 61 years for males and 64 years for females, compared to 73 years for males and 80 years for females in more developed countries. ${ }^{2}$

Gender issues in human immunodeficiency virus (HIV) prevention and care have been on the research agenda for some time. Of concern is the growing gender imbalance of HIV-infection - where now more women than men are HIV-positive in sub-Saharan Africa - that has been documented in many African countries. ${ }^{3-5}$ When it comes to highly active antiretroviral therapy (HAART), Nicastri and colleagues, who completed several reviews of sex/gender-based differences in HAART outcomes, mostly in developed countries, concluded that it is important to conduct gender-sensitive HAART outcome studies in order to determine the sources of male-female outcome differences; specifically whether these differences are the result of biological sex differences or socially-defined gender differences. ${ }^{6,7}$ 
While studies have been undertaken in developing countries to look at differences in mortality between males and females, these studies have not focused on patients accessing treatment in rural and remote areas. Overall, these studies have found that females on HAART have a lower risk of mortality than males. These include studies in Malawi, ${ }^{8,9}$ Cameroon, ${ }^{10}$ Nigeria, ${ }^{11}$ Botswana, ${ }^{12}$ and South Africa. ${ }^{13}$ In contrast to studies in Africa, studies from Brazil showed the opposite result where there was a significant increased risk of mortality for female HAART patients compared to male patients. ${ }^{14,15}$ Finally, a survey of HAART programs in low-income (Africa, Asia, and South America) and high-income (North America and Europe) countries found that overall in both settings there was an increased risk of mortality for males. ${ }^{16}$

Other gender differences in HIV treatment programs have also been reported. For example, gender differences in ART treatment outcomes have been reported in one study undertaken in Uganda and Zimbabwe, where females were found to have better ART outcomes then men. ${ }^{17}$ In developed countries, the findings are mixed. Better treatment outcomes for females have been reported in the United States of America (US), ${ }^{6}$ Switzerland, ${ }^{18}$ and the United Kingdom (UK), ${ }^{19}$ while no differences in outcomes have been observed in other studies from Italy ${ }^{7}$ and the UK. ${ }^{20}$ In addition, adverse reactions to HAART are reported more frequently and consistently in women compared to men from US, Canada, and Botswana. ${ }^{21-24}$ Gender differences in health-seeking behavior for HAART services have also been described in various countries such as Brazil ${ }^{14}$ and from several southern African countries where women who received HAART were younger and had a slower progression to clinical AIDS than men. ${ }^{25}$ Women were reported to be less adherent to HAART than men in Canada, US, and Italy. ${ }^{7,26,27}$ Health providerrelated gender differences were found in two areas of North America where women were less likely to receive a protease inhibitor containing HAART regimen or newly developed antiretroviral (ARV) drugs. ${ }^{28,29}$ Women on HAART in one part of the US were less likely to be referred to a hospital than men when HIV-related complications would have justified hospital treatment. ${ }^{30}$

To date, most research on mortality and treatment outcomes in sub-Saharan Africa have focused on urban areas. In order to investigate treatment outcomes in rural areas in sub-Saharan Africa, our research team evaluated the experiences of patients undergoing treatment between 2006 and 2007 in the Kabarole District in Western Uganda. An estimated 22,400 individuals with HIV-infection reside in this predominantly rural district. ${ }^{31} \mathrm{HIV}$ prevalence in the district is $11.6 \%,{ }^{31}$ which is above the national average of $6.4 \% .^{32}$ In 2006, our team, in conjunction with the Kabarole Health District, implemented a communitybased antiretroviral therapy (CBART) program in Rwimi sub-county located within the Kabarole district. The primary objective of the intervention was to improve access to HAART for persons living with HIV/AIDS living in rural and remote areas. Results from the CBART study were compared with results from a nearby hospital-based HAART program. Though we did not have a specific gender emphasis in the establishment of the CBART program and in how we recruited the cohorts, we expected that bringing treatment closer to where rural populations live would increase access to treatment for women.

The purpose of our study was to examine whether gender differences existed in the outcomes of HAART, specifically all-cause mortality after six months of treatment for HIV patients in rural Western Uganda, and to identify factors associated with mortality in this population.

\section{Methods}

\section{Study population}

This was a prospective cohort study where patients enrolled on HAART were followed-up by our team for six months after starting on HAART. Our study sample consisted of treatment-naïve HIV patients who sequentially presented at either the CBART or hospital-based clinic with the intent to start on combination HAART. The first line treatment consisted of stavudine, lamivudine, and nevirapine (or efavirenz for patients also on rifampicin). Selection criteria for the both cohorts were: aged 18 years or older, treatment naïve, and eligible for HAART according to Ugandan guidelines. ${ }^{33}$ In addition, the CBART cohort had to accept being visited weekly by a community volunteer and be willing to identify a treatment partner. Enrolment for both inception cohorts started on February 2006. By May 2006 the hospital cohort had been recruited $(n=200)$ and by April 2007 the CBART cohort had been recruited ( $n=185$ ); these sample sizes were based on numbers that we required to assess differences in treatment outcomes between the two cohorts for our original study objectives. Though the original study was not established to assess gender differences, we undertook a series of post-hoc subanalyses to look at these differences in more detail as a result of findings from our original study ${ }^{34}$ where we observed a significant difference in treatment outcomes between genders. With a sample size of 385 , the study had adequate 
power of $80 \%$ with $5 \%$ level of significance to detect a $13 \%$ difference in the proportion of survival between males and females at the end of the follow-up.

\section{Data collection}

Baseline clinical and demographic data were obtained from the patient charts for all enrolled patients. Patient clinical data was collected using similar clinical forms to ensure comparability of the data. Demographic data included gender, marital status, occupation, and education. Clinical data included CD4+ cell counts (using a FACS Calibur machine; Becton Dickinson, Nairobi, Kenya). All laboratory tests were carried out in the laboratory of the Joint Clinical Research Centre (JCRC) in Fort Portal, Uganda. This center is part of a network of laboratories in Uganda that uses internationally recognized procedures for maintaining quality control and ensuring reliability of results. The same protocols were used for laboratory tests for both the CBART and hospital-based cohorts to ensure comparability and reliability. Mortality data were obtained by following up patients that had missed more than two visits in the hospital-based cohort and from community volunteers who identified deaths during their weekly patient visits in the CBART cohort. This study considered mortality from all causes.

Statistical procedures included descriptive, univariate, and multivariate analysis. Data from both hospital and community cohorts were pooled for these analyses. Significance of the differences in the demographic and clinical characteristics was tested using the chi-squared tests for categorical variables and the Mann-Whitney $U$ test for continuous variables. Associations between mortality and gender were determined using survival analysis (Kaplan-Meier survival curves) and Cox proportional hazards regression. We used a purposeful selection of predictor variables for the final multivariate analysis and included either variables that were tested to be significant at $P \leq 0.20$ in univariate analyses ${ }^{35}$ or which were pre-determined to be clinically important. $P$-values were two-sided and those $\leq 0.05$ were considered to be statistically significant. We tested the proportional hazard assumption by plotting scaled Schoenfeld residuals for each of the covariate against time and by including an interaction term with time for each covariate. ${ }^{35}$ Martingale residuals were plotted against each of the continuous variables to test the linearity assumption. ${ }^{35}$ Age appeared to violate the linearity assumption and was considered as a categorical variable in the Cox regression model. Overall fit of the model was assessed by plotting log-cumulative hazard plots of Cox-Snell residuals against the log-transformed Cox-Snell residuals. A straight line relationship with unit slope and intercept zero between log-cumulative hazard function of Cox-Snell residuals and log-transformed Cox-Snell residuals indicated that Cox-Snell residuals have an unit exponential distribution and the model-fit was satisfactory. ${ }^{36}$ Statistical analyses were performed using Stata software (version 10.0; Stata Corp, College Station, TX). ${ }^{37}$

\section{Study approval and ethical considerations}

The University of Alberta's Health Research Ethics Board (Panel B) provided ethical approval for the study. In Uganda, approval for the study was obtained from the Uganda National Council of Science and Technology and by the ethical review committee of the School of Public Health, Makerere University, Kampala, Uganda. Within Kabarole District, the study was approved by the District Health Officer and the political representative of Rwimi sub-county (Local Council 3). Each participant was informed about the study in both written and verbal formats and provided written consent.

\section{Results}

A total of 385 patients were recruited into the study, of which $222(57.7 \%)$ were female. Overall, there were more females than males due to higher proportion of females seeking treatment Baseline data and changes in CD4+ cell counts for those in the study have been summarized in Table 1. A lower proportion of females were married compared to males $(31.5 \%$ vs $54.0 \% ; P<0.01)$ with a higher proportion of females being widowed, divorced or separated $(48.0 \%$ for females vs $20.9 \%$ for males; $P<0.01$ ). In addition, a higher proportion of females did not have had any education compared to males $(32.4 \%$ vs $19.1 \% ; P<0.01)$ and a smaller proportion of females had completed secondary level education compared to males ( $11.9 \%$ vs $24.1 \% ; P<0.01)$. A higher proportion of females were unemployed (36.2\% for females vs. $12.3 \%$ for males; $P<0.01$ ) and fewer females were engaged in business or employed in a professional capacity compared to males $(25.7 \%$ vs $44.4 \% ; P<0.01)$. Finally, females had higher baseline CD4+ cell counts than males (median of 147 cells $/ \mu \mathrm{L}$ vs 120 cells $/ \mu \mathrm{L} ; P<0.01$ ). Adherence on HAART was measured by pill counts in the CBART group and was found to be high for both genders; after six months, females had taken $98.5 \%$ of their required doses while males had taken $98.1 \%$ of their required doses (results not shown).

In our unadjusted analyses, $9.0 \%$ of all female patients died compared to $13.5 \%$ of male patients, but this difference was not statistically significant $(P=0.16)$. This corresponded to 
Table I Demographic and clinical information of HIV patients $(n=385)$

\begin{tabular}{|c|c|c|c|}
\hline Demographic variable & Male $N=163(42.3 \%)$ & Female $N=222(57.7 \%)$ & $P$-value \\
\hline \multicolumn{4}{|l|}{ Age group, $n$ (\%) } \\
\hline$<35$ years & $76(46.6 \%)$ & $117(52.7 \%)$ & $0.24 *$ \\
\hline $35+$ years & 87 (53.4\%) & 105 (47.3\%) & \\
\hline \multicolumn{4}{|l|}{ Marital status, $n$ (\%) } \\
\hline Single & $4 I(25.1 \%)$ & $44(20.5 \%)$ & $0.29 *$ \\
\hline Married & $88(54.0 \%)$ & $69(31.5 \%)$ & $<0.0 I^{*}$ \\
\hline Widowed/Divorced/Separated & $34(20.9 \%)$ & $92(48.0 \%)$ & $<0.01 *$ \\
\hline \multicolumn{4}{|l|}{ Educational status, $\mathbf{n}(\%)$} \\
\hline None & $31(19.1 \%)$ & 7I (32.4\%) & $<0.01 *$ \\
\hline Primary & $92(56.8 \%)$ & $122(55.7 \%)$ & $0.83^{*}$ \\
\hline Secondary or above & $39(24.1 \%)$ & $26(11.9 \%)$ & $<0.01 *$ \\
\hline \multicolumn{4}{|l|}{ Occupation, n (\%) } \\
\hline None/Housewife & $20(12.3 \%)$ & $79(36.2 \%)$ & $<0.01 *$ \\
\hline Nonprofessional/Farmer & $70(43.2 \%)$ & $83(38.1 \%)$ & $0.27^{*}$ \\
\hline Business person/Professional & 72 (44.4\%) & $56(25.7 \%)$ & $<0.0$ I* \\
\hline Baseline CD4 (cells/ $\mu \mathrm{l})$, median (IQR) & $120(58-183)$ & $147(82-206)$ & $<0.01 * *$ \\
\hline All-cause mortality deaths & $22(13.5 \%)$ & $20(9.0 \%)$ & $0.16 *$ \\
\hline AIDS-related deaths (in 100 person years) & 31.7 & 20.6 & \\
\hline
\end{tabular}

Notes: $* \chi^{2}$ test; **Mann-Whitney test.

20.6 deaths per 100 person-years for females compared to 31.7 per 100 person-years for males. The differences in mortality are displayed in Kaplan-Meier survival plots (Figure 1).

The survival curves for male and female HIV-infected patients show that in the first five weeks, mortality was almost equal in both sexes. Between five and 25 weeks, mortality was substantially different. In both cohorts, most deaths occurred in the early parts of the treatment phase. The effect size in the univariate analysis was a $35 \%$ difference in mortality in female patients compared to male patients (hazard ratio $[\mathrm{HR}]=0.65 ; P=0.17)$.

In univariate Cox regression analyses (Table 2), baseline CD4+ cell counts, marital status and education levels met the $P \leq 0.20$ significance level for consideration in the multivariate model. Age and sex were forced into the multivariate model based on a-priori assumptions of the importance of these variables. The multivariate Cox regression analysis of the pooled data (Table 2) showed that females were less likely to die (adjusted HR $=0.55,95 \%$ confidence interval [CI]: 0.28-1.07), with the differences being almost statistically significant $(P=0.08)$. Both primary education, compared to having no education (adjusted HR $=0.46,95 \%$ CI: $0.23-0.84 ; P=0.01$ ), and a higher baseline CD4+ cell count (adjusted HR 0.95, 95\% CI: 0.92-0.99; $P=0.02$ ) were predictors of better survival. We did not observe any significant interactions in the multivariate model.
We also analyzed data from both cohorts separately to assess if gender had any relationship with mortality in the different delivery models for HAART. Similar to the pooled data, female patients had a tendency to have lower mortality compared to male patients, though this was not statistically significant (hospital cohort females adjusted HR $=0.45$, 95\% CI: $0.15-1.36 ; P=0.16$, community-based females adjusted HR $=0.61,95 \%$ CI: $0.25-1.49 ; P=0.28$ ) (results not shown).

Residual analyses were carried as described in the methods. Only one of the covariates, education, violated the proportional hazard assumption and was considered as a stratifying variable in the final Cox regression mode but the overall conclusion on gender differences was not affected. There was almost a straight line relationship with unit slope and intercept zero between log-cumulative hazard function of Cox-Snell residuals and log-transformed Cox-Snell residuals indicating that the overall model fit was adequate.

\section{Discussion}

Our study provides information on the relationship between gender and mortality for patients on HAART in a rural area of Western Uganda. An important finding from this study is that female HIV-infected patients on HAART showed a tendency towards lower mortality after adjusting for baseline demographic variables and CD4+ cell count. 


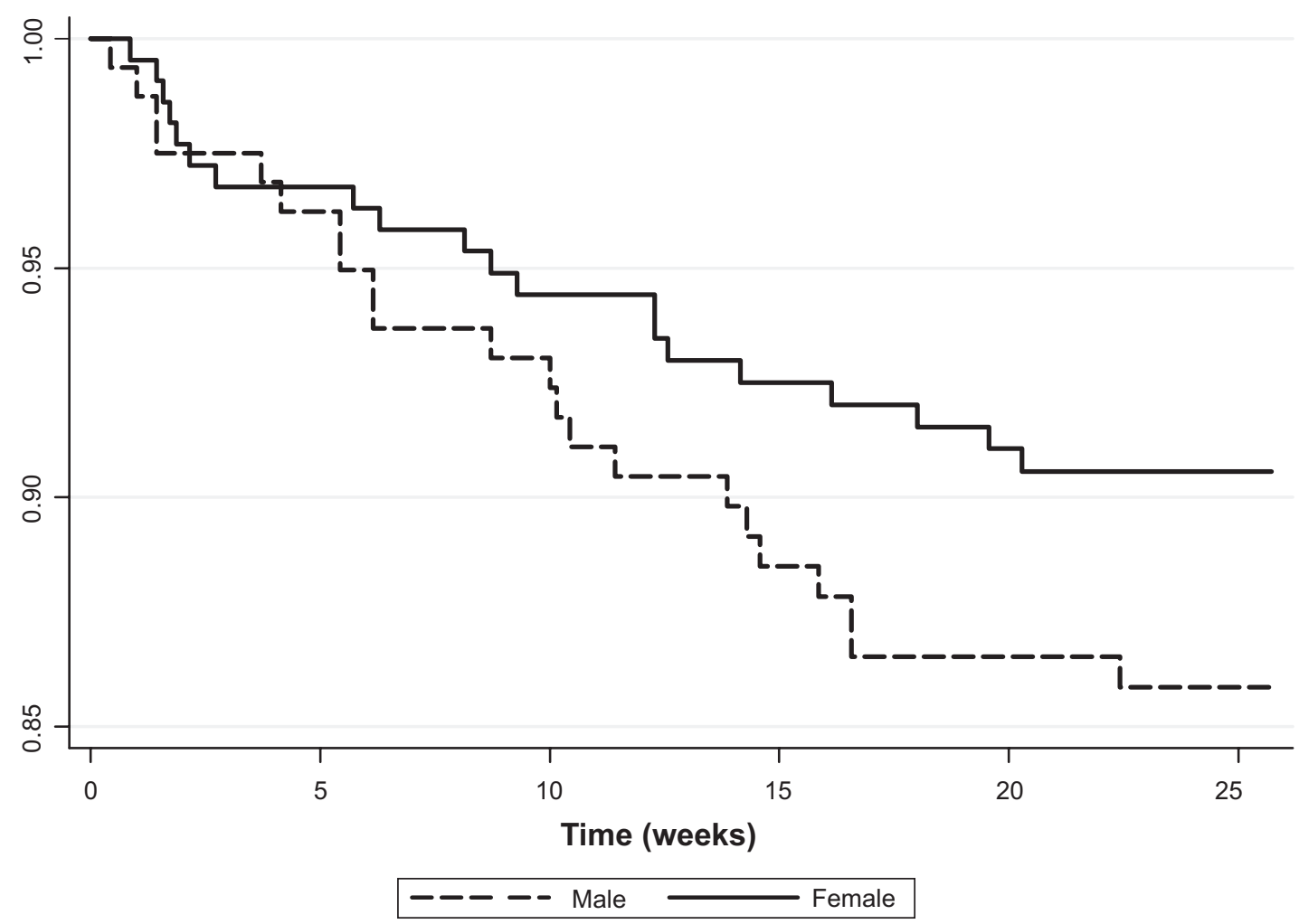

Figure I Kaplan-Meier survival curve for males and females $(P=0.16$ from log-rank test).

Table 2 Hazard ratios (HRs) and 95\% confidence intervals $(95 \% \mathrm{Cls})$ from Cox's regression for factors associated with nonaccidental AIDS-related death

\begin{tabular}{|c|c|c|c|c|}
\hline \multirow[t]{2}{*}{ Variable } & \multicolumn{2}{|c|}{ Univariate analysis } & \multicolumn{2}{|c|}{ Multivariate analysis } \\
\hline & HR (95\% Cl) & $P$-value & HR (95\% Cl) & $P$-value \\
\hline \multicolumn{5}{|l|}{ Age group } \\
\hline$<35$ years & 1.00 & & 1.00 & \\
\hline $35+$ years & $0.89(0.49,1.64)$ & 0.72 & $0.92(0.47, \mid .8 I)$ & 0.82 \\
\hline \multicolumn{5}{|l|}{ Sex } \\
\hline Male & 1.00 & & 1.00 & \\
\hline Female & $0.65(0.36,1.20)$ & 0.17 & $0.55(0.28,1.07)$ & 0.08 \\
\hline \multicolumn{5}{|l|}{ Marital status } \\
\hline Single & 1.00 & & 1.00 & \\
\hline Married & $0.49(0.23,1.04)$ & 0.07 & $0.5 \mathrm{I}(0.23,1.12)$ & 0.09 \\
\hline Widowed/Divorced/Separated & $0.56(0.26,1.19)$ & 0.13 & $0.68(0.30,1.53)$ & 0.30 \\
\hline \multicolumn{5}{|l|}{ Education } \\
\hline None & 1.00 & & 1.00 & \\
\hline Primary & $0.47(0.24,0.93)$ & 0.03 & $0.42(0.21,0.84)$ & 0.01 \\
\hline Secondary or above & $0.61(0.24,1.55)$ & 0.30 & $0.45(0.17,1.21)$ & 0.11 \\
\hline Occupation & 1.00 & & & \\
\hline \multicolumn{5}{|l|}{ No occupation } \\
\hline Nonprofessional/Farmer & $0.86(0.4 \mathrm{I}, \mathrm{I} .8 \mathrm{I})$ & 0.70 & - & \\
\hline Professional/Businessman & $0.79(0.35,1.75)$ & 0.56 & - & \\
\hline Baseline CD4+ cell count* & $0.95(0.91,0.99)$ & 0.01 & $0.95(0.92,0.99)$ & 0.02 \\
\hline
\end{tabular}

Note: *For 10 units increase in CD4+ cell count. 
Our analysis of gender differences within each of the treatment models (CBART vs hospital-based) found that the trend towards a lower risk of mortality for females remained. The effect size and the direction of the effect suggest that females fare better than males regardless of whether they were part of a rural treatment program or a semi-urban program and show that the association between gender and mortality in HAART patients may be independent of factors that are usually associated with rural vs semi-urban populations (eg, cultural and socioeconomic differences). That outcomes are not worse in females is encouraging given that females tend be disadvantaged in terms of education and occupation and being more likely to be widowed, separated or divorced.

Our study findings are in line with studies in other sub-Saharan African countries that looked at gender differences in mortality for patients on HAART. ${ }^{8-12}$ In Malawi, one study found a significant better survival of female HIVinfected patients on HAART compared to male patients (HR 1.70 for males). ${ }^{8}$ A study in Cameroon found a similar higher risk of mortality for men (HR 1.73 for males). ${ }^{10}$ Studies in Nigeria, Botswana, and another in Malawi also found that male gender was associated with higher mortality, though these differences were not statistically significant..$^{9,11,12}$ In South Africa, male gender was associated with higher early mortality (odds ratio [OR] 2.00), and lower later mortality (OR 0.58) compared to females, though the latter findings were not statistically significant. ${ }^{13}$ In a large multi-center study of mortality, researchers found that females had lower mortality than males in both low-income settings (HR 0.84) as well as high-income settings (HR 0.85), though these differences were not statistically significant. Another study in a high income country, Ireland, found again that there were no statistically significant differences in mortality between males and females. ${ }^{38}$ In contrast to these findings, two studies in Brazil have found that females have a higher risk of mortality compared to men (HRs of $3.50^{15}$ and $1.86^{14}$ ) with these differences being statistically significant. With the exception of the Brazil studies, all other study findings corroborate our findings. However, it is important to note that our study is one of the few that evaluate gender differences in mortality for patients living in rural areas of sub-Saharan Africa.

One of the explanations suggested for the gender difference in survival is the observation that female HIV-infected patients enter HAART programs at an earlier stage of their HIV infection than men, likely through their participation in prevention of mother-to-child transmission (PMTCT) programs. ${ }^{8}$ In our cohorts, women tended to seek treatment earlier as apparent by the higher CD4+ cell counts at the time of treatment. The DART trial conducted in Uganda and Zimbabwe show that female HAART patients had better treatment outcomes, as measured by viral suppression, than male patients which would it make reasonable to expect that their survival was probably better as well. ${ }^{17}$ Unfortunately, the authors of the DART study did not present mortality data for male and female patients separately.

An important finding from our study is that female patients had a significantly higher CD4+ cell count at baseline compared to male patients and that the CD4+ cell count was a strong predictor of mortality. This makes it very possible that the lower mortality in our female patients is likely explained, at least in part, by the higher CD4+ cell count at the time of enrollment on HAART. This also indicates that females may access HAART earlier than males. This finding is in line with other studies from sub-Saharan Africa. ${ }^{8,25}$ HIV-positive pregnant women have more opportunities for diagnosis and treatment at an earlier stage in their HIV infection as they access antenatal care. ${ }^{39}$ A recent study from Gugulethu, South Africa identifies the importance of early treatment and that a late start of HAART has disadvantages in terms if higher mortality and that this disadvantage can persist for years. ${ }^{40}$ Increased rates of mortality due to the late initiation of treatment have also been reported by Brinkhof and colleagues. ${ }^{41}$ The second significant association in the multivariate model between a lower HAART mortality and a better educational status is no surprise, and highlights again the importance of education on achieving better health, as shown in numerous studies. In our study, rates of mortality were observed to be similar between males and females in the first five weeks after starting treatment, with the differences becoming more apparent between 5-20 weeks after starting treatment. This suggests that the additional benefits of early access to treatment are realized in the later stages of antiretroviral treatment. In our study, six-month adherence was high and similar between males and females, thus adherence to treatment would not explain the observed differences in mortality.

To the best of our knowledge, the only reported biological differences between male and female HIV-infected patients on HAART are the more frequent adverse reactions to HAART in female HIV-infected patients. Boulassel and colleagues and Wester and colleagues report a higher incidence of lactic acidosis in female HIV-infected patients on HAART as compared to male HIV-infected patients on HAART. ${ }^{23,24}$ In addition, Boulassel and colleagues found that hypersensitivity reactions to ARVs were more likely 
to happen in female HIV-infected patients. ${ }^{23}$ The differentiation between sex and gender factors impacting HAART outcomes and mortality is important, because biologicalbased differences would be difficult to correct, but behavioral and social gender inequities in the health care delivery of HAART services could be addressed effectively by health care systems.

\section{Limitations}

Our sample consisted of patients who actively sought treatment on their own based on a personal decision. Hospital or health center patients are not necessarily representative of all HIV-infected patients in need of treatment and may be different in their characteristics compared to those who do not attend health facilities. This may limit the generalizability of the study results. Finally, our study sample sizes in both cohorts were small, and thus the study had limited power to detect a statistically significant difference in mortality between male and female patients.

\section{Conclusions}

Our study shows that, as in the rest of sub-Saharan Africa, female HIV-patients in Western Uganda on HAART have lower mortality rates than male patients, though this is not conclusive. Evidence from sub-Saharan Africa suggests that women seek care earlier, and hence have lower mortality, is most likely a result of HAART programs being linked with maternity care for women. On the other hand, men seem to delay seeking treatment and enter into treatment at a more advanced stage of HIV infection. This may imply HAART programs and the associated positive outcomes of HAART are more readily accessible to women. If true, this suggests the need for more to be done to ensure that HIV infected men seek treatment at an earlier stage of their HIV infection.

\section{Acknowledgments}

We thank Jean Kipp for her useful comments on the first draft of the paper. The study was financed by the Canadian Institutes of Health Research (CIHR) through grant No. MOP-74586. The authors report no conflicts of interest in this work.

\section{References}

1. Mathers CD, Sadana R, Salomon JA, Murray CJL, Lopez AD. Healthy life expectancy in 191 countries, 1999. Lancet. 2001;357(9269): 1685-1691.

2. US Census Bureau. Global Population Profile: 2002. Washington, DC: US Government Printing Office; 2004.
3. UNAIDS. AIDS epidemic update: November 2009. Geneva, Switzerland: Joint United Nations Programme on AIDS; 2009.

4. Baghdadi G. Gender and medicines: An international public health perspective. $J$ Womens Health. 2005;14(1):82-86.

5. Ojikutu BO, Stone VE. Women, inequality, and the burden of HIV N Engl J Med. 2005;352(7):649-652.

6. Nicastri E, Leone S, Angeletti C, et al. Sex issues in HIV-1-infected persons during highly active antiretroviral therapy: A systematic review. J Antimicrob Chemother. 2007;60(4):724-732.

7. Nicastri E, Angeletti C, Palmisano L, et al. Gender differences in clinical progression of HIV-1-infected individuals during long-term highly active antiretroviral therapy. AIDS. 2005;19(6):577-583.

8. Chen SCC, Yu JKL, Harries AD, et al. Increased mortality of male adults with AIDS related to poor compliance to antiretroviral therapy in Malawi. Trop Med Int Health. 2008;13(4):513-519.

9. Zachariah R, Fitzgerald M, Massaquoi M, et al. Risk factors for high early mortality in patients on antiretroviral treatment in a rural district of Malawi. AIDS. 2006;20(18):2355-2360.

10. Sieleunou I, Souleymanou M, Schonenberger AM, Menten J, Boelaert M. Determinants of survival in AIDS patients on antiretroviral therapy in a rural centre in the Far-North Province, Cameroon. Trop Med Int Health. 2009;14(1):36-43.

11. Desilva MB, Merry SP, Fischer PR, Rohrer JE, Isichei CO, Cha SS. Youth, unemployment, and male gender predict mortality in AIDS patients started on HAART in Nigeria. AIDS Care. 2009;21(1): 70-77.

12. Mujugira A, Wester CW, Kim S, Bussmann H, Gaolathe T. Patients with advanced HIV type 1 infection initiating antiretroviral therapy in Botswana: Treatment response and mortality. AIDS Res Hum Retroviruses. 2009;25(2):127-133.

13. Lawn SD, Myer L, Harling G, Orrell C, Bekker LG, Wood R. Determinants of mortality and nondeath losses from an antiretroviral treatment service in South Africa: Implications for program evaluation. Clin Infect Dis. 2006;43(6):770-776.

14. Braga PE, Cardoso MRA, Segurado AC. Gender differences among persons with HIV admitted to a university reference center in Sao Paulo, Brazil. Cadernos De Saude Publica. 2007;23(11):2653-2662.

15. Santoro-Lopes G, Harrison LH, Moulton LH, et al. Gender and survival after AIDS in Rio de Janeiro, Brazil. J Acquir Immune Defic Syndr Hum Retrovirol. 1998;19(4):403-407.

16. Braitstein P, Brinkhof MWG, Dabis F, et al. Mortality of HIV-1-infected patients in the first year of antiretroviral therapy: comparison between lowincome and high-income countries. Lancet. 2006;367(9513):817-824.

17. Kaleebu P, Pillay D, Walker AS, et al. Virological response to a triple nucleoside/nucleotide analogue regimen over 48 weeks in HIV-1infected adults in Africa. AIDS. 2006;20(10):1391-1399.

18. Wolbers M, Battegay M, Hirschel B, et al. CD4(+) T-cell count increase in HIV-1-infected patients with suppressed viral load within 1 year after start of antiretroviral therapy. Antivir Ther. 2007;12(6):889-897.

19. Porter K, Walker S, Hill T, et al. Changes in outcome of persons initiating highly active antiretroviral therapy at a CD4 count less than 50 cells/mm(3). J Acquir Immune Defic Syndr. 2008;47(2): 202-205.

20. Smith CJ, Sabin CA, Youle MS, et al. Response to efavirenz-containing regimens in previously antiretroviral-naive HIV-positive patients the role of gender. J Acquir Immune Defic Syndr. 2007;46(1):62-67.

21. Tedaldi EM, Absalon J, Thomas AJ, Shlay JC, van den Berg-Wolf M. Ethnicity, race, and gender-Differences in serious adverse events among participants in an antiretroviral initiation trial: Results of CPCRA 058 (FIRST study). J Acquir Immune Defic Syndr. 2008;47(4):441-448.

22. Heath KV, Chan KJ, Singer J, O'Shaughnessy MV, Montaner JSG, Hogg RS. Incidence of morphological and lipid abnormalities: gender and treatment differentials after initiation of first antiretroviral therapy. Int J Epidemiol. 2002;31(5):1016-1020.

23. Boulassel MR, Morales R, Murphy T, Lalonde RG, Klein MB. Gender and long-term metabolic toxicities from antiretroviral therapy in HIV-1 infected persons. J Med Virol. 2006;78(9):1158-1163. 
24. Wester CW, Okezie OA, Thomas AM, et al. Higher-than-expected rates of lactic acidosis among highly active Antiretroviral therapy-treated women in Botswana - Preliminary results from a large randomized clinical trial. J Acquir Immune Defic Syndr. 2007;46(3):318-322.

25. Braitstein P, Boulle A, Nash D, et al. Gender and the use of antiretroviral treatment in resource-constrained settings: Findings from a multicenter collaboration. J Womens Health. 2008;17(1):47-55.

26. Kuyper LM, Wood E, Montaner JSG, Yip B, O’Connell JM, Hogg RS. Gender differences in HIV-1 RNA rebound attributed to incomplete antiretroviral adherence among HIV-infected patients in a populationbased cohort. J Acquir Immune Defic Syndr. 2004;37(4):1470-1476.

27. Holstad MKM, Pace JC, De AK, Ura DR. Factors associated with adherence to antiretroviral therapy. J Assoc Nurses AIDS Care. 2006;17(2):4-15.

28. Reif S, Whetten K, Thielman N. Association of race and gender with use of antiretroviral therapy among HIV-infected individuals in the southeastern United States. South Med J. 2007;100(8):775-781.

29. Furler MD, Einarson TR, Walmsley S, Millson M, Bendayan R. Longitudinal trends in antiretroviral use in a cohort of men and women in Ontario, Canada. AIDS Patient Care STDs. 2006;20(4):245-257.

30. Floris-Moore M, Lo YT, Klein RS, et al. Gender and hospitalization patterns among HIV-Infected drug users before and after the availability of highly active antiretroviral therapy. J Acquir Immune Defic Syndr. 2003;34(3):331-337.

31. Kabarole District. District Information Portal. Available from: http:// www.kabarole.go.ug/. Accessed August 12, 2008.

32. Uganda Ministry of Health. Uganda HIV/AIDS sero-behavioral survey 2004-2005. Kampala, Uganda: Uganda Ministry of Health; 2006.
33. Uganda Ministry of Health. National antiretroviral treatment and care guidelines for adults and for children. 1st Edition. Kampala, Uganda: Uganda Ministry of Health; 2003.

34. Kipp W, Konde-Lule J, Saunders LD, et al. Results of a communitybased antiretroviral treatment program for HIV-1 infection in western Uganda. Curr HIV Res. 2010 Feb 18. [Epub ahead of print].

35. Hosmer D, Lemeshow S, May S. Applied Survival Analaysis. Regression Modeling of Time-to-Event Data (2nd Edition). Hoboken, NJ: John Wiley \& Sons Inc.; 2008.

36. Collett D. Modelling Survival Data in Medical Research. London, UK: Chapman and Hall; 1994.

37. STATA Statistical Software: Release 10.0 [computer program]. College Station, TX: Stata Corporation; 2001

38. Dunne MT, Ruskin HJ, Mulcahy FM. Survival with AIDS in Ireland. AIDS. 1997;11(10):1281-1290.

39. Okong P. HIV/AIDS and women's health in Uganda: Lingering gender inequity. J Obstetr Gynaecol Canada. 2006;28:980-982.

40. Lawn S, Little F, Bekker L, et al. Changing mortality risk associated with CD4 cell response to long-term ART: sub-Saharan Africa. Montreal, Canada: Paper presented at Sixteenth Conference on Retroviruses and Opportunistic Infections; 2009.

41. Brinkhof M, Boulle A, Weigel R, et al. Mortality of HIV-infected patients starting ART: Comparisons with the general population in Southern Africa. Montreal, Canada: Paper presented at: Sixteenth Conference on Retroviruses and Opportunistic Infections; 2009.
International Journal of Women's Health

\section{Publish your work in this journal}

The International Journal of Women's Health is an international, peerreviewed open-access journal publishing original research, reports, reviews and commentaries on all aspects of women's healthcare including gynaecology, obstetrics, and breast cancer. Subject areas include: Chronic conditions (migraine headaches, arthritis, osteoporosis);

\section{Dovepress}

Endocrine and autoimmune syndromes; Sexual and reproductive health; Psychological and psychosocial conditions. The manuscript management system is completely online and includes a very quick and fair peer-review system. Visit http://www.dovepress.com/ testimonials.php to read real quotes from published authors. 